\title{
Atrial Dissociation
}

\author{
Nobuharu Akatsuka, M.D., Toshiko Maekawa, M.D., \\ and Masayasu Hrraoka, M.D.*
}

\section{SUMmaRY}

Atrial dissociation was recorded in a patient with malignant hypertension and microangiopathic hemolytic anemia. Digitalis intoxication was considered the cause of atrial dissociation.

\section{Additional Indexing Words:}

Digitalis intoxication Diaphragmatic movement

7 THE phenomenon of so-called "atrial dissociation" in animal experi1 ments was first shown at the beginning of this century. ${ }^{11}$ However, the actual occurrence and the observation of similar electrocardiographic phenomena in clinical practice have been a matter of controversy. There have been several extensive reviews of this phenomenon either supporting ${ }^{21,3)}$ or denying ${ }^{4)}$ the reported cases. Those who did not support the concept believed that most reported phenomena could be attributed to artifacts of extracardiac origin. ${ }^{4,5)}$ Actually several authors showed that these artifacts could simulate the electrocardiographic appearance of atrial dissociation. ${ }^{4-8)}$ However, granting these situations could occur in some clinical settings, their arguments do not exclude the possibility that electrical events similar to the one observed by Hering ${ }^{11}$ may occur in some limited area of the human atrium. More observations and careful analysis are necessary to clarify this problem.

We report a case in which, during the recording, sources of artifacts were eliminated as much as possible. The ECG tracing obtained from this patient could reasonably be interpreted as atrial dissociation.

\section{Case Report}

A 37-year-old male was admitted to the University Hospital of Tokyo on January 16, 1973 because of hypertension and hemorrhagic diathesis. He was known to have hypertension since 1970 and took anti-hypertensive medication at irregular intervals. The high blood pressure was left uncontrolled until November Japan.

From the First Department of Internal Medicine, University of Tokyo, Hongo, Bunkyo-ku, Tokyo,

Department of Cardiovascular Diseases, Medical Research Institute, Tokyo Medical and Dental University, Yushima, Bunkyo-ku, Tokyo, Japan.

Received for publication October 27, 1975. 
1972, when he noticed headache and difficulty in stopping bleeding after shaving. The blood pressure at that time was 250/150. The urinalysis revealed a marked proteinuria, 1-5 red cells and 10-20 white cells per high power field. BUN was $24.3 \mathrm{mg} / 100 \mathrm{ml}$ and creatinine was $18.5 \mathrm{mg} / 100 \mathrm{ml}$. Thrombocytopenia was also noticed (thrombocytes: $6.5 \times 10^{4}$ ). Hospitalization was advised for the control of blood pressure and hemorrhagic diathesis.

After admission, he was treated with alpha-methyldopa, chlortharidone, and reserpine, and the blood pressure was controlled within the range of 160 to 180 systolic and 100 to 120 diastolic. His hospital course was complicated by a recurrent fever of unknown etiology lasting for about 40 days, which was not much affected by the administration of aminobenzyl-penicillin or chloramphenicol. His renal function and thrombocytopenia also deteriorated gradually during hospitalization. From February 27, 1973 to March 11, he suffered from frequent attacks of hiccoughs, which were difficult to control at first but subsided spontaneously later.

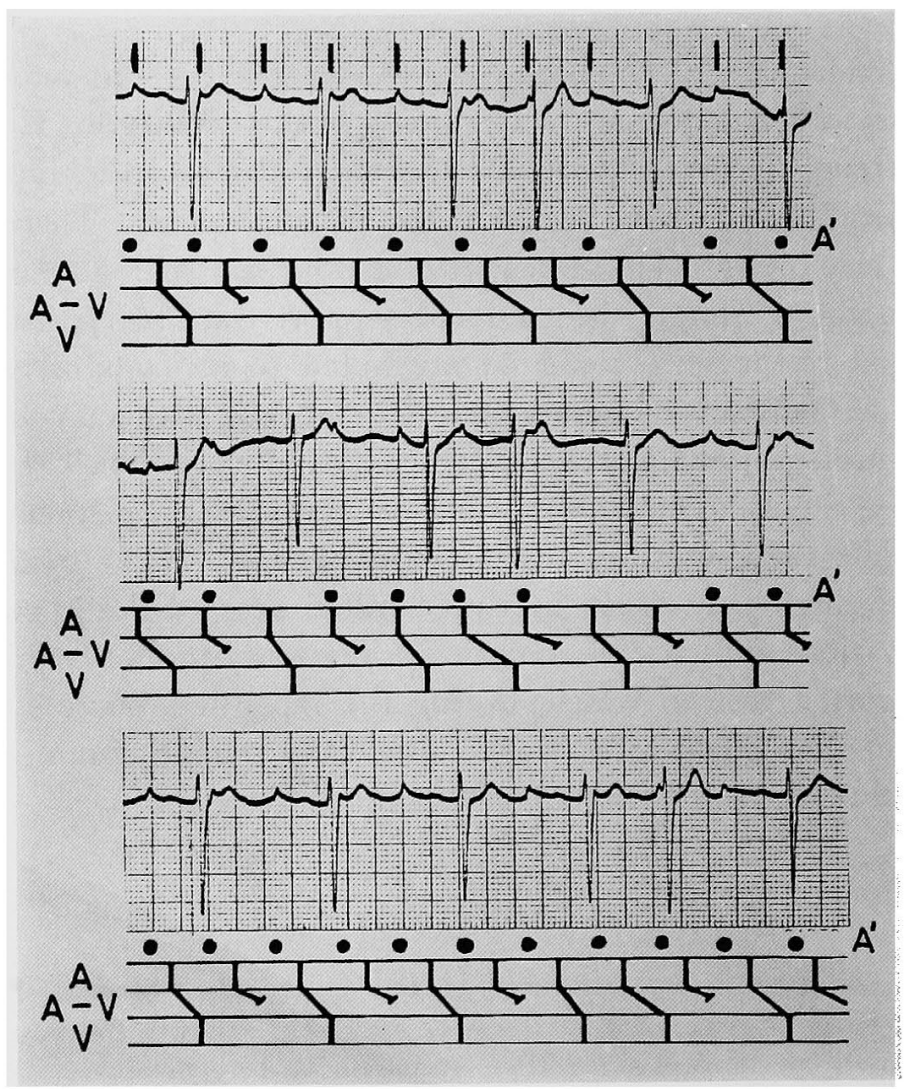

Fig. 1. Electrocardiographic recording and ladder gram of the arrhythmia. Three rows represent the continuous recording of lead $\mathrm{V}_{2}$. Ectopic $\mathrm{P}^{\prime}$-wave-like deflections are indicated by the vertical lines on the top row and are marked by $\mathrm{A}^{\prime}$ in the ladder gram. $\mathrm{A}^{\prime}$ never reached the ventricle. Sinus $\mathrm{P}$ waves showed 2:1 or $3: 1 \mathrm{~A}-\mathrm{V}$ block with Wenckebach phenomenon indicated by progressive lengthening of $\mathrm{A}-\mathrm{V}$ conduction time followed by the block. 
On March 13, 1973, he developed acute pulmonary edema, which was treated with lanatoside $\mathrm{C}$ and furosemide. BUN at that time was $61 \mathrm{mg} / 100 \mathrm{ml}$. Because of his renal failure and cardiac failure, frequent peritoneal dialysis was performed, with moderate improvement. His cardiac condition stayed about the same and the administration of digitalis and diuretics was continued.

On May 13, 1973, he developed arrhythmia without any distress. The ECG revealed supraventricular arrhythmia, 2nd degree A-V block with Wenckeback phenomenon and occasional aberrant ventricular conduction. $\mathrm{P}^{\prime}$ wave-like deflection ( $\mathrm{P}^{\prime}$ wave) with the rate of $130 / \mathrm{min}$ was recorded in $\mathrm{V}_{2}$ (Fig. 1). To eliminate the possible contamination by extracardiac electrical activity, all the electrical machines were disconnected from the patient's bed at this moment. At the same time careful observations on the patient's chest and abdominal wall were made but no vigorous diaphragmatic movements nor any tic-like movement of any muscle were noted. The patient was not in respiratory distress at that time. $\mathrm{P}^{\prime}$ wave was only recorded in lead $\mathrm{V}_{2}$ but not in any other leads recorded at the same time. The repeated ECG recording 2 hours after the initial one showed PAT with 2: 1 block and did not show any $\mathrm{P}^{\prime}$ wave deflections. The diagnosis of digitalis intoxication was made and the administration of digitalis was discontinued.

Thrombocytopenia and severe hypertension continued and renal failure deteriorated gradually. He died on June 8, 1973 from cerebral bleeding. The clinical diagnosis was malignant hypertension and thrombotic thrombocytopenic purpura, and the pathological diagnosis was malignant hypertension with microangiopathic hemolytic anemia.

\section{DisGussion}

Atrial dissociation is considered to arise when an area of atrial tissue is completely surrounded by a border of bidirectional block (i.e. entrance and exit block), so that an ectopic impulse activating an enclosed area is unable to disturb the sinus rhythm or to activate the ventricle. The electrocardiographic characteristics of atrial dissociation are: 1) presence of an ectopic atrial rhythm independent of the basic rhythm (sinus or ectopic), 2) the impulses of the atrial rhythm are not conducted to the ventricles, 3) ectopic atrial rhythm is commonly a slow rhythm but occasionally it may be a tachycardia, flutter or fibrillation. In some reported cases, $\mathrm{P}^{\prime}$ waves were followed by oscillations. Most patients who showed atrial dissociation were critically ill with congestive heart failure or distressed respiration and died shortly after an attack of atrial dissociation. Only a few cases were followed up for fairly long periods. ${ }^{9), 10)}$ Because of the rather short duration of an attack of atrial dissociation and also because of the severity of the basic diseases, investigations other than the conventional twelve lead ECG are usually not attempted. Therefore, the diagnosis of atrial dissociation is usually made exclusively from a single conventional ECG. Thus, the diagnosis inevitably contains some uncertainty. 
On the other hand, there have been a number of convincing reports which did not support most of the clinically diagnosed atrial dissociation.4),5) In these reports, $\mathrm{P}^{\prime}$ waves were interpreted as the electrical phenomenon related to the vigorous diaphragmatic movement, such as hiccough, distressed respiration, and tic, clonus, or flutter of diaphragm. Oscillations following $\mathrm{P}^{\prime}$ wave were also recorded in these conditions. Higgins et al $^{4)}$ asserted that most electrocardiograms reported as atrial dissociation were artifacts due to strenuous respiratory movement and to electromyograms of the accessory respiratory muscles. According to their analysis, $\mathbf{P}^{\prime}$ waves are usually 40 to $60 / \mathrm{min}, 0.2 \mathrm{mV}, 0.03 \mathrm{sec}$ duration followed by the vibration of 500 to $3,000 / \mathrm{min}$, and the $\mathrm{P}^{\prime}$ waves are recorded only in II, III, and $\mathrm{aV}_{\mathrm{F}}$. Aygen et $\mathbf{a}^{(6)}$ showed experimentally the origin of the $\mathrm{P}^{\prime}$ waves in the diaphragmatic muscles. The rate of the spastic movements of respiratory muscles ranges from 60 to $300 / \mathrm{min}$. The higher rates of 200 to $300 / \mathrm{min}$ are said to be observed only in instances of spasm of the diaphragm. ${ }^{7}$ Inspection and palpation of vibratory movements of the chest and the abdominal wall are most helpful in the diagnosis of diaphragmatic tic."

In 1 reported case, an intracardiac ECG was recorded together with a conventional ECG.8) The intracardiac ECG did not show a $\mathrm{P}^{\prime}$ wave, and, therefore, the $\mathrm{P}^{\prime}$ wave in the conventional EGG was considered to be an action potential of the muscle of diaphragm.

In the present case, the rate of $\mathrm{P}^{\prime}$ waves was $130 / \mathrm{min}$ and faster than the basic sinus rhythm, and the $\mathrm{P}^{\prime}$ waves were recorded only in $\mathrm{V}_{2}$ lead and not in II, III, and $\mathrm{aV}_{\mathrm{F}}$, and were not followed by an oscillation of high frequency. Although the patient had repeated attacks of hiccough 2 months prior to the time when atrial dissociation was noticed, there was no abnormality in respiratory movement of the thorax during the arrhythmia attack. All the other possibilities of artifacts which might give rise to a $\mathbf{P}^{\prime}$ wave-like deflection in an $\mathrm{ECG}^{2)}$ were eliminated. In our case, $\mathbf{P}^{\prime}$ wave was recorded in only $V_{2}$, probably because the area excited by this ectopic focus was small and the mean $\mathrm{P}^{\prime}$ vector was directed along the $\mathrm{V}_{2}$ lead axis.

Most reported cases were not in the state of digitalis intoxication. ${ }^{3)}$ In our case, the patient showed arrhythmias probably due to digitalis intoxication during and shortly after the cessation of atrial dissociation (cf; 2nd degree A-V block of Wenckebach phenomenon, PAT with block) and these arrhythmias disappeared after discontinuation of digitalis.

Recent animal experiments disclosed the possible mechanism of digitalisinduced arrhythmia. The first sign of digitalis intoxication observed at the cellular level was a transient depolarization of transmembrane potential in the specialized conducting tissues. ${ }^{11,12)}$ This transient depolarization can 
reach threshold and cause one or more automatic responses. Therefore digitalis can induce ectopic focus activity by this mechanism as well as by increasing phase 4 depolarization. ${ }^{13)}$ Moreover, it was shown that conduction block was easily associated with this transient depolarization as well. ${ }^{14}$ ) Therefore, in digitalis intoxication, an area which shows the transient depolarization becomes not only a site of ectopic impulse formation but also a region of conduction block, and this will promote the appearance of special forms of arrhythmia such as atrial dissociation. It can reasonably be predicted, therefore, that this may occur in the digitalized human atrium. We consider that this patient showed an attack of atrial dissociation together with other arrhythmias due to digitalis intoxication.

\section{ACKNowledgements}

The authors wish to thank Professor Michael F. Wilson and Dr. Mary L. Morgan of West Virginia University, Morgantown, West Virginia, U.S.A., for checking the English text.

\section{References}

1. Hering $\mathrm{HD}$ : Zur experimentellen Analyse der unregelmaessigkeiten des Herzschlages. Arch Gcs Physiol 82: 1, 1900

2. Cohen J, Scherf D: Complete interatrial and intraatrial block (dissociation). Am Heart J 70: 23, 1965

3. Cihung EK: A reappraisal of atrial dissociation. Am J Cardiol 28: 111, 1971

4. Higgins TG, Phillips JH, Summer RG: Atrial dissociation. An electrocardiographic artifact produced by the accessory muscles of respiration. Am J Cardiol 18: 132, 1966

5. Fleischmann P, Bar-Khayim Y: Electromyogram of respiratory muscles registered in the ordinary electrocardiogram. Brit Heart J 30: 242, 1968

6. Aygen MM, Manoach M, Liberman UA, Mintz U, Savransky H: Diaphragmatic action potentials registered in the elcctrocardiogram. A clinical and experimental study. Am Heart J 83: 630, 1972

7. Dressler W, Kleinfeld M: Tic of the respiratory muscles. Report of three cases and review of literature. Am J Med 16: 61, 1954

8. Soler-Soler J, Angel-Ferrer J: Complete atrial dissociation versus diaphragmatic action potentials. Brit Heart J 36: 452, 1974

9. Igarashi M, Katayama F, Hinohara S: Two cases of atrial dissociation. Am J Cardiol 11: 267,1963

10. Clark WH, Douglas JE: Atrial dissociation-fact or artifact? J Electrocardiol 6: 373, 1973

11. Ferrier GR, Saunders JH, Mendez C: Cellular mechanism for the generation of ventricular arrhythmia by acetylstrophanthidin. Circulat Res 32:600, 1973

12. Hashimoto K, Moe GK: Transient depolarization induced by acetylstrophanthidin in specialized tissue of dog atrium and ventricle. Circulat Res 32: 618, 1973

13. Vassalle M, Karis J, Hoffman BF: Toxic effects of ouabain on Purkinje fibers and ventricular muscle fibers. Am J Physiol 203: 433, 1962

14. Saunders JH, Ferrier GR, Moe GK: Conduction block associated with transient depolarizations induced by acetylstrophanthidin in isolated canine Purkinje fibers. Circulat Res 32: 610,1973 